\title{
Expression of MUC2, MUC5AC, MUC5B, and MUC6 mucins in colorectal cancers and their association with the CpG island methylator phenotype
}

Michael D Walsh ${ }^{1,2}$, Mark Clendenning ${ }^{1}$, Elizabeth Williamson ${ }^{3}$, Sally-Ann Pearson ${ }^{1}$, Rhiannon J Walters ${ }^{1}$, Belinda Nagler ${ }^{1}$, David Packenas ${ }^{1}$, Aung K Win ${ }^{3}$, John L Hopper ${ }^{3}$, Mark A Jenkins ${ }^{3}$, Andrew M Haydon ${ }^{4}$, Christophe Rosty ${ }^{1,5,6}$, Dallas R English ${ }^{3,7}$, Graham G Giles ${ }^{3,7}$, Michael A McGuckin ${ }^{8}$, Joanne P Young ${ }^{1}$ and Daniel D Buchanan ${ }^{1}$

${ }^{1}$ Cancer and Population Studies Group, Queensland Institute of Medical Research, Herston, QLD, Australia;

${ }^{2}$ Department of Histopathology, Sullivan Nicolaides Pathology, Taringa, QLD, Australia; ${ }^{3}$ Centre for MEGA Epidemiology, School of Population Health, University of Melbourne, Melbourne, VIC, Australia;

${ }^{4}$ Department of Medical Oncology, Alfred Hospital, Prahran, VIC, Australia; ${ }^{5}$ Royal Brisbane and Women's Hospital, University of Queensland Centre for Clinical Research, Herston, QLD, Australia; ${ }^{6}$ Envoi Pathology, Herston, QLD, Australia; ${ }^{7}$ Cancer Epidemiology Centre, Cancer Council Victoria, Carlton, VIC, Australia and ${ }^{8}$ Mater Medical Research Institute, Mater Misericordiae Hospital, South Brisbane, QLD, Australia

Mucinous differentiation is associated with both $\mathrm{CpG}$ island methylator phenotype and microsatellite instability in colorectal cancer. The mucinous phenotype derives from abundant expression of the colonic goblet cell mucin, MUC2, and de novo expression of gastric foveolar mucin, MUC5AC. We, therefore, investigated the protein expression levels of MUC2 and MUC5AC, as well as MUC5B and MUC6, in molecular subtypes of colorectal cancer. Seven-hundred and twenty-two incident colorectal carcinomas occurring in 702 participants of the Melbourne Collaborative Cohort Study were characterized for methylator status, MLH1 methylation, somatic BRAF and KRAS mutations, microsatellite-instability status, MLH1, MSH2, MSH6, and PMS2 mismatch repair, and p53 protein expression, and their histopathology was reviewed. Protein expression levels of MUC2, MUC5AC, MUC5B, MUC6, and the putative mucin regulator CDX2 were compared with molecular and clinicopathological features of colorectal cancers using odds ratios and corresponding $95 \%$ confidence intervals. MUC2 overexpression ( $>25 \%$ positive tumor cells) was observed in $33 \%$ colorectal cancers, MUC5B expression in 53\%, and de novo MUC5AC and MUC6 expression in $50 \%$ and $39 \%$, respectively. Co-expression of two or more of the mucins was commonly observed. Expression of MUC2, MUC5AC and MUC6 was strongly associated with features associated with tumorigenesis via the serrated neoplasia pathway, including methylator positivity, somatic BRAF p.V600E mutation, and mismatch repair deficiency, as well as proximal location, poor differentiation, lymphocytic response, and increased $T$ stage (all $\boldsymbol{P}<0.001)$. Overexpression was observed in tumors with and without mucinous differentiation. There were inverse associations between expression of all four mucins and p53 overexpression. CDX2 expression was inversely associated with MUC2, MUC5AC and MUC6 expression. Our results suggest that, in methylator-positive tumors, mucin genes on chromosome 11p15.5 region undergo increased expression via mechanisms other than direct regulation by CDX2.

Modern Pathology (2013) 26, 1642-1656; doi:10.1038/modpathol.2013.101; published online 28 June 2013

Keywords: CIMP; colorectal cancer; methylation; MUC2; MUC5AC; MUC5B; MUC6

Correspondence: Dr MD Walsh, PhD, Cancer and Population Studies Group, Queensland Institute of Medical Research, 300 Herston Road, Herston, QLD 4006, Australia.

E-mail: Michael.Walsh@qimr.edu.au

Received 25 January 2013; revised 2 May 2013; accepted 3 May 2013; published online 28 June 2013
Colorectal cancer displays considerable molecular and biological heterogeneity, which is due to, at least in part, differing pathways of neoplastic progression. The majority of colorectal cancer arises through the adenoma-carcinoma sequence 
characterized by chromosomal instability with associated accumulation of genetic alterations in tumor-suppressor genes, such as APC and TP53. ${ }^{1}$ Colorectal carcinoma also arises from atypical serrated polyps, demonstrating little evidence of chromosomal instability. Rather, tumor-suppressor genes are inactivated by widespread epigenetic silencing known as CpG Island Methylator Phenotype (CIMP) ${ }^{2}$ often accompanied by the somatic BRAF p.V600E mutation. ${ }^{3-5}$ CIMP colorectal cancers are associated with clinicopathological features such as proximal location, poor grade, presence of tumor-infiltrating lymphocytes, and mucinous differentiation, as well as frequently demonstrating molecular somatic events including the BRAF p.V600E mutation and high levels of microsatellite instability, but with much lower levels of TP53 mutation than their chromosomal unstable colorectal carcinoma counterparts. ${ }^{6-12}$

Mucins are high-molecular weight proteins characterized by the presence of large amino acid tandem repeat sequences that show allelic size variation. Secreted or gel-forming mucins comprise five known types: MUC2, MUC5AC, MUC5B, MUC6, and MUC19. All but MUC19 are encoded by genes present in a cluster on chromosome 11p15. ${ }^{13}$ MUC2 is the predominant secreted mucin synthesized by colonic goblet cells, ${ }^{14}$ along with MUC5B. ${ }^{15}$ In the normal colon, MUC5AC is rarely expressed, and only by a minority of goblet cells, and there are conflicting reports concerning the expression of MUC6 in the colon. ${ }^{16,17}$ MUC5B expression in the lower gastrointestinal tract is relatively unexplored and appears to be restricted to colonic goblet cells. ${ }^{15} \mathrm{We}$ and others have shown that MUC2 and MUC5AC mucins are expressed at high levels in mucinous colorectal cancers and by tumors exhibiting microsatellite instability. ${ }^{18-21}$ CIMP colorectal cancers also exhibit significant mucinous differentiation, suggesting that one or more gel-forming mucins are overexpressed in CIMP. Several regulatory mechanisms governing mucin gene expression have been demonstrated, including promoter $\mathrm{CpG}$ island methylation ${ }^{22,23}$ and activation of the EGFR-RAS-RAF signal transduction pathway. ${ }^{24}$ In addition, the regulation of mucin gene expression by various transcription factors such as Sp1, AP-1, and CDX2 has been explored in a range of tissues (reviewed by Andrianifahanana et $a l^{25}$ ). Of these, the homeobox protein, CDX2, reportedly regulates MUC2 expression in gastrointestinal epithelial goblet cells ${ }^{26,27}$ and has shown a loss of expression in microsatellite-unstable colorectal cancers ${ }^{28,29}$ and serrated polyps of the colorectum. ${ }^{30}$

We report here the results from a large series of colorectal carcinomas demonstrating an association between expression of mucins, MUC2, MUC5AC, MUC5B, and MUC6 and the presence of somatic BRAF p.V600E mutation, CIMP, microsatellite instability, and loss of CDX2 expression.

\section{Materials and methods}

\section{Patient Cohort}

Archival tumor samples were obtained from participants enrolled in The Melbourne Collaborative Cohort Study, a prospective study of 41514 people (17 045 males and 24469 females) recruited between 1990 and 1994. Participants were aged between 27 and 81 years at baseline (99.3\% were aged 40-69 years). Full details of the design, recruitment, and study procedures have been published previously. ${ }^{31}$ All participants gave informed consent.

Between study enrollment and December 2010, 976 participants were diagnosed with incident colorectal carcinoma and were ascertained through linkage to the Victorian Cancer Registry to which notification is a legal requirement. Ethical approval was obtained from relevant participating centers and the Queensland Institute of Medical Research Human Research Ethics Committee under protocol P799.

\section{Histopathology Review}

Colorectal carcinomas were reviewed by one of the two specialist gastrointestinal pathologists (Professor Jeremy Jass and Dr Christophe Rosty) with regard to anatomical site, tumor grade, tumor margin, and synchronous colorectal carcinoma. The presence of any minor mucinous component (defined as mucinous differentiation present in $<50 \%$ of the tumor). Tumor budding was assessed using the criteria described by Ueno et $a l^{32}$ ( $\geq 10$ foci of isolated tumor cells or clusters of fewer than five tumor cells at the invasive margin within a $\times 25$ microscopic field). Peritumoral lymphocytes (a mantle or cap of lymphoid cells at the deepest point of direct spread), Crohn's-like lymphocytic reaction (at least three nodular lymphoid aggregates deep to the invasive margin in a $\times 4$ field), and tumor-infiltrating lymphocytes (at least four intraepithelial lymphocytes per $\times 40$ field) were scored using the criteria described previously by Young et al. ${ }^{33}$ Tumors in the ileocecal junction, cecum, ascending colon, hepatic flexure, and transverse colon were grouped as right-sided (proximal) colon cancers (ICD-O-3 codes C180, C182, C183, and C184), ${ }^{34}$ whereas those in the splenic flexure (C185), descending colon (C186), sigmoid colon (C187), recto-sigmoid junction (C199) and rectum (C209) were grouped as left-sided (distal).

\section{Immunohistochemistry}

Tissues for analysis were fixed for several hours in neutral buffered formalin and then embedded in paraffin. Paraffin sections $(4 \mu \mathrm{m})$ were routinely dewaxed and rehydrated, then subjected to heatinduced epitope retrieval in either High $\mathrm{pH}$ Target 
Retrieval solution (Dako, Carpinteria, CA) (MUC2 and MUC6) or Reveal Decloaking solution (BioCare Medical, Concord, MA) for $8 \mathrm{~min}$, and then incubated with primary antibody for $90 \mathrm{~min}$. The antibodies used were (a) anti-MUC2 (clone Ccp58, 1/500 dilution) (Santa Cruz Biotechnology Inc., Santa Cruz, CA), (b) anti-MUC5AC (clone 45M1, 1/750 dilution) (Neomarkers Inc., Fremont, CA), (c) anti-MUC6 (clone CLH5, 1/250 dilution) (Santa Cruz Biotechnology Inc.), (d) anti-MUC5B (clone EU-MUC5B, 1/500 dilution), (e) anti-p53 (clone DO7, 1/100 dilution) (Dako), and (f) anti-CDX2 (clone CDX2-88, 1/100 dilution) (BioCare Medical) followed by the EnVision Plus Mouse HRP detection system (Dako). Antigenic sites were developed using $\mathrm{DAB}+$ liquid chromogen (Dako), and then the sections were counterstained with hematoxylin before mounting. Expression of the MLH1, MSH2, MSH6, and PMS2 was assessed as described previously. ${ }^{35}$

Stained sections were scored by one observer (Dr Michael Walsh) blinded to clinical and molecular testing results, and a subset of tumors was scored independently by another observer (Professor Jeremy Jass) to assess reproducibility. The proportion of positive cancer cell staining was graded as follows: 0 (negative), $<10 \%(1+), 11-25 \%(2+)$, $26-50 \%(3+), 51-75 \%(4+)$, and $>75 \%(5+)$. The staining intensity of cancer cells was graded as weak $(1+)$, moderate $(2+)$, or strong $(3+)$. Note was also made of the cellular localization of stains for each antibody (cytoplasmic membrane, apical membrane, and extracellular membrane). Cases were classified as positive for MUC2 and MUC5B where staining was observed in $>25 \%$ tumor cells, and $>0 \%$ for MUC5AC or MUC6. Histologically normal colonic mucosa served as positive control tissue for MUC2 and MUC5B, and normal stomach as the positive control for MUC5AC and MUC6. Previously demonstrated colorectal cancers were used as positive and negative controls for assessment of p53 overexpression, and normal colonic epithelium served as the CDX2 control.

\section{Molecular Assays}

Colorectal carcinoma DNA from formalin-fixed paraffin-embedded tissue was tested for microsatellite instability using a 10-marker microsatellite panel as previously described. ${ }^{36}$ Tumors were scored as showing microsatellite instability when instability was detected at $>40 \%$ of loci tested. ${ }^{37}$ Testing for somatic mutations in KRAS codons 12 and 13 was performed using direct Sanger sequencing as previously described. ${ }^{3}$ Testing for the BRAF p.V600E somatic mutation was performed using an allele-specific PCR assay as described previously. ${ }^{37}$ Positive controls were run in each experiment, and $10 \%$ of samples were replicated with $100 \%$ concordance. Methylation analysis of the CIMP markers RUNX3, SOCS1, CACNA1G, NEUROG1, and IGF2 and the MLH1 promoter was performed on bisulphite-converted tumor DNA using MethyLight as previously described. ${ }^{3,37}$ High levels of CIMP (CIMP-positive) were defined when $\geq 3$ of the 5 markers were positively methylated, whereas cancers with $<3$ positively methylated markers were considered CIMP-negative.

\section{Statistical Analysis}

The association between mucin expression and clinicopathological features was assessed using $\chi^{2}$-tests and quantified using odds ratios (ORs) with $95 \%$ confidence intervals (CIs). Differences between mean ages at diagnosis were assessed between groups using $t$-test after assessing the equality of variances. The level of statistical significance was set at $P<0.05$.

\section{Results}

Altogether, 976 incident cases of colorectal carcinoma were identified, and for $722(74 \%)$ of these, tissue was available. Ten participants had two and one had three synchronous colorectal cancers, whereas two had two metachronous cancers, and a further two had three metachronous cancers. Patient ( $n=702,367$ males, 335 females) age at colorectal cancer diagnosis ranged from 42.0 to 83.4 years (mean age $=67.9$ years, s.d. 7.9). Details of clinicopathological and molecular features of the tumors are summarized in Supplementary Table 1. There was considerable overlap in clinicopathological features associated with both CIMP-positive and $B R A F$ mutation, including mucinous differentiation (Supplementary Table 2).

\section{MUC2 Expression}

Positive staining for MUC2 core protein using Ccp58 is characterized by intense cytoplasmic reactivity in goblet cells in normal colonic epithelium (Figure 1a). The majority of cancers (67\%) exhibited either complete negativity for MUC2 (8\%) or rare $(<25 \%)$ stained epithelial cells $(59 \%)$ with or without apparent goblet cell differentiation (Figure 1b). Using a threshold of $\geq 25 \%$ of tumor cells stained, 215/655 (33\%) colorectal cancers were classified as MUC2-positive. The associations between MUC2 expression and tumor clinicopathological features are presented in Table 1. MUC2positive carcinomas were twice as likely to be proximal $(\mathrm{OR}=2.83,95 \% \mathrm{CI}=2.01-3.99)$, and have mucinous differentiation $(P<0.001)$, although strong MUC2 expression could be observed in areas with conventional adenocarcinoma differentiation (Figure 1c). MUC2 expression was also associated with higher $\mathrm{T}$ stage $(\mathrm{OR}=1.64,95 \% \mathrm{CI}=1.09-2.47)$, 
and the presence of tumor-infiltrating lymphocytes $(\mathrm{OR}=2.43,95 \%$ CI 1.66-3.58) and Crohn's-like aggregates $(\mathrm{OR}=2.30, \quad 95 \% \quad \mathrm{CI}=1.49-3.56)$. All signet ring cell tumors and 49/51 (96\%) of mucinous tumors showed MUC2 positivity (Figure 1d).

MUC2-positive colorectal cancers were more likely to be CIMP-positive (51/75, 68\%) than CIMP-negative $(145 / 539,27 \%) \quad(\mathrm{OR}=5.77, \quad 95 \%$ $\mathrm{CI}=3.43-9.72)$, more frequently $B R A F$ p.V600Emutated $(\mathrm{OR}=3.77,95 \% \mathrm{CI}=2.48-5.73)$, demonstrate $M L H 1$ promoter methylation $(\mathrm{OR}=4.74,95 \%$ $\mathrm{CI}=2.73-8.26$ ), show DNA mismatch repair deficiency, that is loss of expression of one or more mismatch repair proteins $(\mathrm{OR}=7.15, \quad 95 \%$ $\mathrm{CI}=4.28-11.95)$, and were less likely to have p53 overexpression $(\mathrm{OR}=0.42,95 \% \mathrm{CI}=0.30-0.60)$.

\section{MUC5AC Expression}

MUC5AC was present in the foveolar epithelial cells of the normal stomach control sections both as cytoplasmic staining and also extracellular mucin (Figure 1e). In the normal colon distant from the tumor, MUC5AC was not expressed, but normal epithelium in close proximity to cancers frequently showed staining of a variable proportion of normal goblet cells, predominantly staining of the thecal contents but also extracellular mucin (Figure 1f). Any MUC5AC staining of tumor cells was considered de novo expression, and 321/649 (49\%) cancers were classified as MUC5AC-positive. Expression ranged from infrequent isolated cells or clusters of cells (Figure 1g) to diffuse positivity (Figure 1h).

Tumor clinicopathological features that are associated with MUC5AC expression are presented in Table 1. As was observed for MUC2 expression, MUC5AC-positive colorectal carcinomas were more likely to be proximal $(\mathrm{OR}=1.98,95 \% \mathrm{CI}=1.42-$ $2.75)$, have mucinous differentiation $(P<0.001)$, be of higher $\mathrm{T}$ stage $(\mathrm{OR}=1.57,95 \% \mathrm{CI}=1.08-2.28)$, and have tumor-infiltrating lymphocytes $(\mathrm{OR}=2.21$, 95\% CI = 1.51-3.24). MUC5AC expression was also associated with higher-grade carcinomas $(\mathrm{OR}=2.24$, $95 \% \mathrm{CI}=1.51-3.34)$ and female sex $(\mathrm{OR}=1.48,95 \%$ $\mathrm{CI}=1.08-2.01)$. Positive MUC5AC expression was associated with CIMP positivity $(\mathrm{OR}=8.58,95 \%$ $\mathrm{CI}=4.18-17.63), \quad B R A F \quad$ p.V600E mutation $(\mathrm{OR}=4.08,95 \% \mathrm{CI}=2.56-6.50)$, MLH1 promoter methylation $(\mathrm{OR}=6.36,95 \% \mathrm{CI}=3.16-12.79)$, and DNA mismatch repair deficiency $(\mathrm{OR}=6.65,95 \%$ $\mathrm{CI}=3.59-12.30)$ and was less likely to have p53 overexpression $\quad(\mathrm{OR}=0.52, \quad 95 \% \quad \mathrm{CI}=0.38-0.72)$.
The association between MUC2 and MUC5AC expression, and CIMP and BRAF status is shown in a 'heat map' (Figure 2).

\section{MUC5B Expression}

There was intense goblet cell reactivity for MUC5B in normal colon, which was most prominent in the basal two-thirds of crypts in all specimens, diminishing to little or no MUC5B staining of surface epithelium (Figure 3a). In tumors, staining was localized to the cytoplasm, commonly in the supranuclear region, as well as extracellular mucin. There was often intense reactivity on the apical cell membranes in gland spaces within tumors (Figures 3b-d). The relationship between MUC5B expression and tumor clinicopathological features is summarized in Table 2. MUC5B-expressing carcinomas were associated with female gender $(\mathrm{OR}=2.28$, 95\% CI = 1.07-4.84), mucinous differentiation $(P=0.006)$, and the presence of tumor-infiltrating lymphocytes $(\mathrm{OR}=2.62,95 \% \mathrm{CI}=1.08-6.36)$, but showed no statistical evidence of increased prevalence in the proximal colon. In addition, associations with mismatch repair deficiency $(\mathrm{OR}=4.17$, $95 \% \mathrm{CI}=1.43-12.16)$ and lack of p53 expression $(\mathrm{OR}=0.24,95 \% \mathrm{CI}=0.11-0.51)$ and MUC5B expression were observed.

\section{MUC6 Expression}

MUC6 was present in the gastric gland epithelial cells of the normal stomach control sections as cytoplasmic staining (Figure $3 \mathrm{e}$ ) and was not seen in normal colon epithelium. Staining in a subset of colorectal cancers was exclusively cytoplasmic in nature, and any staining of tumor cells was considered de novo expression. Of the 126 colorectal carcinomas tested, 49 (39\%) showed evidence of MUC6 staining. In the majority of MUC6-positive cases, staining was restricted to $<10 \%$ tumor cells (31/49, 63\%) (Figures 3f-h). Tumor clinicopathological features that are associated with MUC6 expression are presented in Table 2. Positive staining for MUC6 was associated with proximal location $(\mathrm{OR}=4.83,95 \% \mathrm{CI}=2.20-10.56)$, presence of tumor-infiltrating lymphocytes $\quad(\mathrm{OR}=7.71, \quad 95 \%$ $\mathrm{CI}=3.05-19.46)$, and mucinous differentiation $(P=0.029)$. As for MUC2 and MUC5AC expression, MUC6 expression was associated with CIMP-positive colorectal carcinomas $(\mathrm{OR}=1.33, \quad 95 \% \quad \mathrm{CI}$ =1.09-1.63), BRAF p.V600E mutation $(\mathrm{OR}=7.85$,

Figure 1 (a) MUC2 expression restricted to the cytoplasm of goblet cells in normal colonic epithelium. (b) Tumor scored negative for MUC2, showing only isolated cells $(<25 \%)$ stained for MUC2. These cells have typical goblet cell differentiation. (c) Extensive MUC2 immunoreactivity in an adenocarcinoma without any mucinous differentiation. (d) Strong MUC2 staining in a mucinous carcinoma. (e) MUC5AC reactivity in the foveolar epithelium of normal stomach. (f) Moderate staining of a typical adenocarcinoma with intense apical cytoplasmic reactivity. (g) Isolated MUC5AC-positive tumor cells with evidence of staining of extracellular mucin. The transitional epithelium adjacent to the tumor is strongly stained, but more distant colonic epithelium is essentially MUC5AC-negative. (h) Intense MUC5AC staining of a colorectal carcinoma including staining of goblet cell contents and extracellular mucin. 

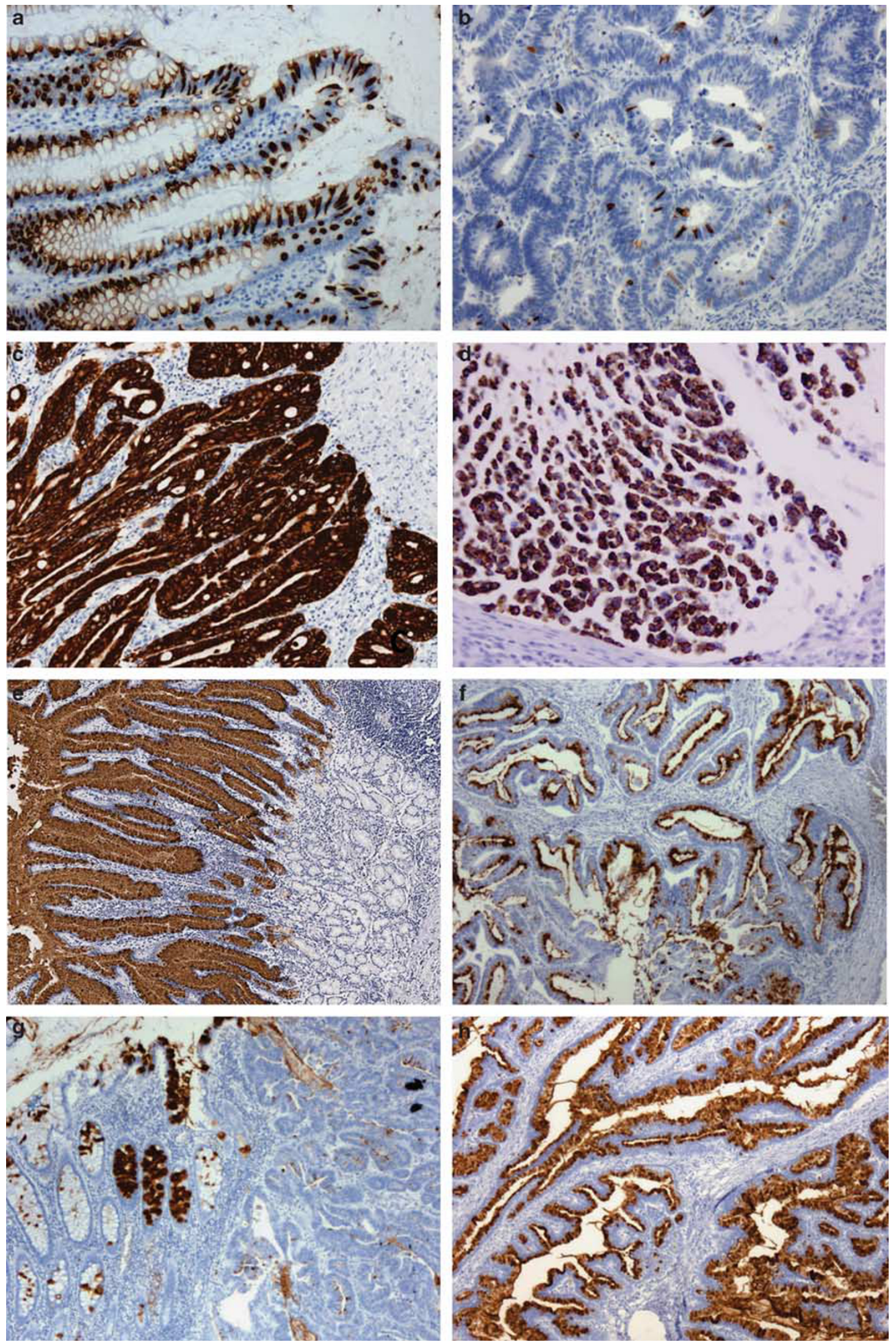

Figure 1 For caption see page 1645. 
Table 1 MUC2 and MUC5AC expression related to tumor clinicopathological features

\begin{tabular}{|c|c|c|c|c|c|c|}
\hline & \multicolumn{3}{|c|}{ MUC2 } & \multicolumn{3}{|c|}{ MUC5AC } \\
\hline & $\begin{array}{c}\text { MUC2-positive, } \\
\mathrm{N}(\%)\end{array}$ & P-value & $\begin{array}{c}\text { OR } \\
(95 \% \text { CI) }\end{array}$ & $\begin{array}{c}\text { MUC5AC-positive } \\
\mathrm{N}(\%)\end{array}$ & $\mathrm{P}$-value & $\begin{array}{c}\text { OR } \\
(95 \% \text { CI })\end{array}$ \\
\hline \multicolumn{7}{|l|}{ Gender } \\
\hline Male & $111 / 351(32)$ & 0.505 & 1.12 & $156 / 347(45)$ & 0.015 & 1.48 \\
\hline Female & $104 / 304(34)$ & & $0.81-1.56$ & $165 / 302(55)$ & & $1.08-2.01$ \\
\hline \multicolumn{7}{|l|}{ Ethnicity } \\
\hline Non-mediterranean & 169/502 (34) & 0.429 & 0.84 & $258 / 501(51)$ & 0.073 & 0.70 \\
\hline Mediterranean & $45 / 150(30)$ & & $0.57-1.25$ & $62 / 145(43)$ & & $0.49-1.02$ \\
\hline \multicolumn{7}{|l|}{ Tumor side } \\
\hline Left & $99 / 410(24)$ & $<0.001$ & 2.83 & $180 / 417(43)$ & $<0.001$ & 1.98 \\
\hline Right & $110 / 232(47)$ & & $2.01-3.99$ & $132 / 220(60)$ & & $1.42-2.75$ \\
\hline \multicolumn{7}{|l|}{ Tumor type } \\
\hline Adenocarcinoma & $44 / 416(11)$ & $<0.001$ & & $147 / 420(35)$ & $<0.001$ & \\
\hline $\begin{array}{l}\text { Adenocarcinoma } 1-49 \% \\
\text { mucinous component }\end{array}$ & $113 / 174(65)$ & & & $119 / 167(71)$ & & \\
\hline Mucinous $>50 \%$ & 49/51 (96) & & & $46 / 49(94)$ & & \\
\hline Signet ring cell ca. & $9 / 9(100)$ & & & $8 / 8(100)$ & & \\
\hline Undifferentiated & $0 / 4(0)$ & & & $1 / 4(25)$ & & \\
\hline \multicolumn{7}{|l|}{ Tumor grade } \\
\hline Well/moderate & $168 / 524(32)$ & 0.407 & 1.19 & $235 / 517(45)$ & $<0.001$ & 2.24 \\
\hline Poor/undifferentiated & $47 / 131(36)$ & & $0.79-1.77$ & $86 / 132(65)$ & & $1.51-3.34$ \\
\hline \multicolumn{7}{|l|}{ Tumor T stage } \\
\hline $\mathrm{T} 1 / \mathrm{T} 2$ & $39 / 155(25)$ & 0.022 & 1.64 & $65 / 150(43)$ & 0.018 & 1.57 \\
\hline $\mathrm{T} 3 / \mathrm{T} 4$ & $159 / 448(35)$ & & $1.09-2.47$ & $243 / 445(56)$ & & $1.08-2.28$ \\
\hline \multicolumn{7}{|l|}{ Lymph node metastases } \\
\hline Absent & $105 / 317(33)$ & 0.595 & 1.10 & $162 / 316(51)$ & 0.312 & 1.18 \\
\hline Present & $90 / 254(35)$ & & $0.78-1.57$ & $140 / 252(56)$ & & $0.85-1.66$ \\
\hline \multicolumn{7}{|l|}{ Venous invasion } \\
\hline Absent & $177 / 527(34)$ & 0.225 & 0.78 & $264 / 519(51)$ & 0.277 & 1.19 \\
\hline Present & $21 / 53(40)$ & & $0.46-1.34$ & $42 / 76(55)$ & & $0.74-1.94$ \\
\hline \multicolumn{7}{|l|}{ Margins } \\
\hline Expanding & $142 / 441(32)$ & 0.695 & 1.09 & $219 / 432(51)$ & 0.520 & 1.14 \\
\hline Infiltrating & $55 / 161(34)$ & & $0.75-1.60$ & $88 / 163(54)$ & & $0.80-1.64$ \\
\hline \multicolumn{7}{|l|}{ Budding } \\
\hline Absent & $128 / 368(35)$ & 0.061 & 0.69 & $191 / 370(52)$ & 0.724 & 0.93 \\
\hline Present & $55 / 205(27)$ & & $0.47-1.00$ & $98 / 197(50)$ & & $0.76-1.31$ \\
\hline \multicolumn{7}{|c|}{ Tumor-infiltrating lymphocytes } \\
\hline Absent & $144 / 510(28)$ & $<0.001$ & 2.43 & $226 / 497(45)$ & $<0.001$ & 2.21 \\
\hline Present & $68 / 139(49)$ & & $1.66-3.58$ & $94 / 145(65)$ & & $1.51-3.24$ \\
\hline \multicolumn{7}{|l|}{ Peritumoral lymphocytes } \\
\hline Absent & $168 / 497(34)$ & 0.738 & 0.91 & $253 / 485(52)$ & 0.603 & 0.89 \\
\hline Present & $35 / 110(32)$ & & $0.59-1.42$ & $56 / 114(49)$ & & $0.59-1.33$ \\
\hline \multicolumn{7}{|l|}{ Crohn's-like aggregates } \\
\hline Absent & $147 / 492(30)$ & $<0.001$ & 2.30 & $242 / 480(50)$ & 0.162 & 1.36 \\
\hline Present & $50 / 101(50)$ & & $1.49-3.56$ & $61 / 105(58)$ & & $0.89-2.09$ \\
\hline \multicolumn{7}{|l|}{ p53 Overexpression } \\
\hline Absent & $123 / 281(44)$ & $<0.001$ & 0.42 & $158 / 265(60)$ & $<0.001$ & 0.52 \\
\hline Present & $85 / 343(25)$ & & $0.30-0.60$ & $153 / 353(43)$ & & $0.38-0.72$ \\
\hline \multicolumn{7}{|l|}{$B R A F$} \\
\hline WT & $140 / 518(27)$ & $<0.001$ & 3.77 & $226 / 512(44)$ & $<0.001$ & 4.08 \\
\hline p.V600E & 67/115 (58) & & $2.48-5.73$ & 87/114 (76) & & $2.56-6.50$ \\
\hline
\end{tabular}


Table 1 (Continued)

\begin{tabular}{|c|c|c|c|c|c|c|}
\hline & \multicolumn{3}{|c|}{ MUC2 } & \multicolumn{3}{|c|}{$M U C 5 A C$} \\
\hline & $\begin{array}{c}\text { MUC2-positive, } \\
\mathrm{N}(\%)\end{array}$ & $\mathrm{P}$-value & $\begin{array}{c}\text { OR } \\
(95 \% \text { CI })\end{array}$ & $\begin{array}{c}\text { MUC5AC-positive } \\
\mathrm{N}(\%)\end{array}$ & $\mathrm{P}$-value & $\begin{array}{c}\text { OR } \\
(95 \% \text { CI })\end{array}$ \\
\hline \multicolumn{7}{|l|}{ KRAS } \\
\hline WT & $136 / 453(30)$ & 0.011 & 1.61 & $221 / 456(48)$ & 0.239 & 1.26 \\
\hline Codons $12 / 13$ mutation & $72 / 176(41)$ & & $1.12-2.32$ & 90/166 (54) & & $0.88-1.80$ \\
\hline \multicolumn{7}{|l|}{ Mismatch repair } \\
\hline Proficient & $154 / 569(27)$ & $<0.001$ & 7.15 & $250 / 563(44)$ & $<0.001$ & 6.65 \\
\hline Deficient & $61 / 84(73)$ & & $4.28-11.95$ & $69 / 82(84)$ & & $3.59-12.30$ \\
\hline \multicolumn{7}{|l|}{ CIMP } \\
\hline Negative & $145 / 539(27)$ & $<\mathbf{0 . 0 0 1}$ & 5.77 & $237 / 537(44)$ & $<0.001$ & 8.58 \\
\hline Positive & $51 / 75(68)$ & & $3.43-9.72$ & $61 / 70(87)$ & & $4.18-17.63$ \\
\hline \multicolumn{7}{|l|}{ MLH1 methylation } \\
\hline Negative & $143 / 516(28)$ & $<0.001$ & 4.74 & $229 / 509(45)$ & $<0.001$ & 6.36 \\
\hline Positive & 40/62 (65) & & $2.73-8.26$ & $52 / 62(84)$ & & $3.16-12.79$ \\
\hline
\end{tabular}

Abbreviations: CI, confidence intervals; CIMP, CpG island methylator phenotype; OR, odds ratio; WT, wild type.

The statistically significant $P$-values have been highlighted in bold.

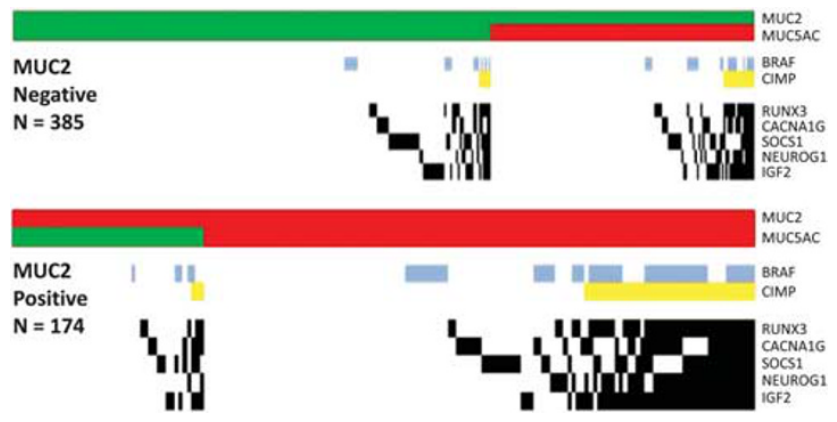

Figure 2 'Heat map' style presentation of the association between MUC2 and MUC5AC positivity, somatic BRAF p.V600E mutation, overall CIMP status, and methylation of individual markers. From the top, MUC2 and MUC5AC expression status is indicated either in red (positive) or green (negative). Blue boxes indicate the presence of BRAF mutation, yellow indicates CIMP-positive cases, and the individual markers RUNX3, CACNA1G, SOCS1, NEUROG1, or IGF2 are indicated in black where methylated.

95\% CI $=2.62-23.53$ ), and DNA mismatch repair deficiency $(\mathrm{OR}=27.38,95 \% \mathrm{CI}=6.02-124.47)$, as well as MLH1 methylation $(\mathrm{OR}=10.63,95 \%$ $\mathrm{CI}=1.18-96.00)$, and was inversely associated with p53 overexpression $(\mathrm{OR}=0.36,95 \% \mathrm{CI}=0.17-0.76)$.

\section{Co-expression of Mucins}

Overall, 117 tumors were stained for all four mucin proteins, and of these, $25(21.4 \%)$ were negative for all four mucins, $29(24.8 \%)$ expressed only one mucin, $21(17.9 \%)$ expressed two mucins, 17 $(14.5 \%)$ expressed three mucins, and 15 (12.8\%) expressed all four mucins. Expression of the four mucins in individual colorectal carcinomas is represented in a heat map (Figure 4). Co-expression of more than two mucins was more common in females $(23 / 47 ; 48.9 \%)$ than males $(19 / 70 ; 27.1 \%)$ $(\mathrm{OR}=2.57,95 \% \mathrm{CI}=1.18-5.60)$, in proximal tumors $(23 / 44 ; 52.3 \%)$ compared with distal cancers (17/69; $24.6 \%)(\mathrm{OR}=3.35,95 \% \mathrm{CI}=1.50-7.50)$, and mismatch repair-deficient cancers (18/22) compared with mismatch repair proficient tumors (24/94) $(\mathrm{OR}=13.13,95 \% \mathrm{CI}=4.04-42.65)$. There were also strong associations between expression of three or four mucins and the presence of tumor-infiltrating lymphocytes $(\mathrm{OR}=7.22,95 \% \mathrm{CI}=2.87-18.18)$ and p53 negativity $(\mathrm{OR}=0.16,95 \% \mathrm{CI}=0.07-0.38)$. High levels of MUC2 or MUC5B expression ( $>50 \%$ tumor cells stained) were each predictive of co-expression of other mucins. Of the 43 high MUC2-expressing tumors, 33 (76.7\%) showed immunoreactivity for at least two other mucins compared with only 9 out of 74 MUC2-low/negative cancers $(\mathrm{OR}=23.83,95 \% \mathrm{CI}=8.83-64.35)$. Similarly, $37 / 50(74 \%)$ of high MUC5B-expressing tumors also demonstrated expression for two or more other mucins, whereas only $5 / 67$ (7.5\%) of MUC5B-low/negative cancers showed similar co-expression ( $\mathrm{OR}=35.29,95 \% \mathrm{CI}=11.64-106.97)$. There were also strong associations between coexpression of three or more mucins and the presence of CIMP (OR $=9.00,95 \% \mathrm{CI}=1.67-48.41)$ and $B R A F$ mutation $(\mathrm{OR}=3.36$, 95\% CI 1.22-9.24).

\section{Mucin Expression and CDX2 Status}

CDX2 immunohistochemistry was performed on a subset of 120 tumors and was scored as absent in 6 cases $(5 \%)$ and reduced $(<50 \%$ tumor nuclei stained) in a further 15 cases $(12.5 \%)$. The associations between reduced or complete loss of expression of CDX2 and expression of MUC2, MUC5AC, MUC5B, and MUC6 are presented in Table 3. 
Table 2 MUC5B and MUC6 expression related to tumor clinicopathological features

\begin{tabular}{|c|c|c|c|c|c|c|}
\hline & \multicolumn{3}{|c|}{$M U C 5 B$} & \multicolumn{3}{|c|}{ MUC6 } \\
\hline & $\begin{array}{c}\text { MUC5B-positive } \\
\text { N (\%) }\end{array}$ & $\mathrm{P}$-value & $\begin{array}{c}\text { OR } \\
(95 \% C I)\end{array}$ & $\begin{array}{c}\text { MUC6-positive, } \\
\text { N (\%) }\end{array}$ & $\mathrm{P}$-value & $\begin{array}{c}\text { OR } \\
(95 \% C I)\end{array}$ \\
\hline \multicolumn{7}{|l|}{ Gender } \\
\hline Male & $32 / 72(44)$ & 0.040 & 2.28 & $26 / 75(35)$ & 0.267 & 1.55 \\
\hline Female & $31 / 48(65)$ & & $1.07-4.84$ & $23 / 51(45)$ & & $0.75-3.21$ \\
\hline \multicolumn{7}{|l|}{ Ethnicity } \\
\hline Non-Mediterranean & 49/92 (53) & 0.667 & 0.82 & 37/98 (38) & 0.825 & 1.13 \\
\hline Mediterranean & $13 / 27(48)$ & & $0.35-1.92$ & $11 / 27(41)$ & & $0.48-2.70$ \\
\hline \multicolumn{7}{|l|}{ Tumor side } \\
\hline Left & $34 / 71(48)$ & 0.343 & 1.49 & $18 / 73(25)$ & $<0.001$ & 4.83 \\
\hline Right & $26 / 45(58)$ & & $0.70-3.16$ & $30 / 49(61)$ & & $2.20-10.56$ \\
\hline \multicolumn{7}{|l|}{ Tumor type } \\
\hline Adenocarcinoma & $27 / 65(42)$ & 0.006 & & $19 / 70(27)$ & 0.029 & \\
\hline Adenocarcinoma 1-49\% & $21 / 33(64)$ & & & $17 / 33(52)$ & & \\
\hline Mucinous $>50 \%$ & 13/16 (81) & & & 9/17 (53) & & \\
\hline Signet ring cell ca. & $2 / 2(100)$ & & & $2 / 2(100)$ & & \\
\hline Undifferentiated & $0 / 3(0)$ & & & $2 / 3(67)$ & & \\
\hline \multicolumn{7}{|l|}{ Tumor grade } \\
\hline Well/moderate & 48/85 (56) & 0.228 & 0.58 & $34 / 90$ (38) & 0.691 & 1.18 \\
\hline Poor/undifferentiated & $15 / 35(43)$ & & $0.26-1.28$ & $15 / 36(42)$ & & $0.54-2.59$ \\
\hline \multicolumn{7}{|l|}{ Tumor T stage } \\
\hline $\mathrm{T} 1 / \mathrm{T} 2$ & $15 / 27(56)$ & 0.659 & 0.78 & $8 / 29(28)$ & 0.189 & 1.93 \\
\hline $\mathrm{T} 3 / \mathrm{T} 4$ & $40 / 81(49)$ & & $0.33-1.87$ & $36 / 85(42)$ & & $0.77-4.84$ \\
\hline \multicolumn{7}{|l|}{ Lymph node metastases } \\
\hline Absent & $31 / 57(54)$ & 0.555 & 0.74 & $26 / 60(43)$ & 0.697 & 0.80 \\
\hline Present & $22 / 47(47)$ & & $0.34-1.60$ & $19 / 50(38)$ & & $0.37-1.72$ \\
\hline \multicolumn{7}{|l|}{ Venous invasion } \\
\hline Absent & $48 / 92(52)$ & 0.359 & 0.69 & 37/96 (39) & 0.584 & 0.96 \\
\hline Present & $6 / 14(43)$ & & $0.22-2.14$ & $6 / 16(38)$ & & $0.32-2.85$ \\
\hline \multicolumn{7}{|l|}{ Margins } \\
\hline Expanding & $31 / 60(52)$ & 0.847 & 0.90 & $24 / 62(39)$ & 1.000 & 1.02 \\
\hline Infiltrating & $23 / 47(49)$ & & $0.42-1.92$ & $20 / 51(39)$ & & $0.48-2.18$ \\
\hline \multicolumn{7}{|l|}{ Budding } \\
\hline Absent & $35 / 62(56)$ & 0.297 & 0.62 & $22 / 64(34)$ & 0.533 & 1.33 \\
\hline Present & $16 / 36(44)$ & & $0.27-1.41$ & $16 / 39(41)$ & & $0.58-3.02$ \\
\hline \multicolumn{7}{|c|}{ Tumor-infiltrating lymphocytes } \\
\hline Absent & $41 / 87(47)$ & 0.035 & 2.62 & $25 / 92(27)$ & $<0.001$ & 7.71 \\
\hline Present & $21 / 30(70)$ & & $1.08-6.36$ & $23 / 31(74)$ & & $3.05-19.46$ \\
\hline \multicolumn{7}{|l|}{ Peritumoral lymphocytes } \\
\hline Absent & $42 / 81(52)$ & 1.000 & 1.05 & $32 / 84(38)$ & 0.547 & 1.30 \\
\hline Present & $18 / 34(53)$ & & $0.47-2.33$ & $16 / 36(44)$ & & $0.59-2.87$ \\
\hline \multicolumn{7}{|l|}{ Crohn's-like aggregates } \\
\hline Absent & $45 / 88(51)$ & 0.797 & 0.85 & $32 / 92(35)$ & 0.017 & 3.75 \\
\hline Present & $8 / 17(47)$ & & $0.30-2.40$ & $12 / 18(67)$ & & $1.29-10.93$ \\
\hline \multicolumn{7}{|l|}{ p53 Overexpression } \\
\hline Absent & 43/62 (69) & $<0.001$ & 0.24 & $31 / 61(51)$ & 0.010 & 0.36 \\
\hline Present & 20/57 (35) & & $0.11-0.51$ & 17/63 (28) & & $0.17-0.76$ \\
\hline \multicolumn{7}{|l|}{$B R A F$} \\
\hline WT & $41 / 82(50)$ & 0.805 & 1.22 & 26/86 (30) & $<0.001$ & 7.85 \\
\hline p.V600E & $11 / 20(55)$ & & $0.46-3.26$ & $17 / 22(77)$ & & $2.62-23.53$ \\
\hline
\end{tabular}


Table 2 (Continued)

\begin{tabular}{|c|c|c|c|c|c|c|}
\hline & \multicolumn{3}{|c|}{$M U C 5 B$} & \multicolumn{3}{|c|}{ MUC6 } \\
\hline & $\begin{array}{c}\text { MUC5B-positive } \\
\text { N (\%) }\end{array}$ & $\mathrm{P}$-value & $\begin{array}{c}\text { OR } \\
(95 \% C I)\end{array}$ & $\begin{array}{c}\text { MUC6-positive } \\
\mathrm{N}(\%)\end{array}$ & $\mathrm{P}$-value & $\begin{array}{c}\text { OR } \\
(95 \% C I)\end{array}$ \\
\hline \multicolumn{7}{|l|}{ KRAS } \\
\hline WT & $39 / 78(50)$ & 0.285 & 1.64 & $36 / 83(43)$ & 0.391 & 0.65 \\
\hline Codons $12 / 13$ mutation & $18 / 29(62)$ & & $0.68-3.91$ & $10 / 30(33)$ & & $0.27-1.57$ \\
\hline \multicolumn{7}{|l|}{ Mismatch repair } \\
\hline Proficient & 44/95 (46) & 0.009 & 4.17 & 28/101 (28) & $<0.001$ & 27.38 \\
\hline Deficient & $18 / 23(78)$ & & $1.43-12.16$ & $21 / 23(91)$ & & $6.02-124.47$ \\
\hline \multicolumn{7}{|l|}{ CIMP } \\
\hline Negative & $35 / 77(45)$ & 0.466 & 2.00 & $24 / 82(29)$ & $<\mathbf{0 . 0 0 1}$ & 1.33 \\
\hline Positive & $5 / 8(63)$ & & $0.45-8.96$ & $8 / 8(100)$ & & $1.09-1.63$ \\
\hline \multicolumn{7}{|l|}{ MLH1 methylation } \\
\hline Negative & $33 / 70(47)$ & 0.200 & 5.61 & $24 / 75(32)$ & 0.020 & 10.63 \\
\hline Positive & $5 / 6(83)$ & & $062-50.48$ & $5 / 6(83)$ & & $1.18-96.00$ \\
\hline
\end{tabular}

Abbreviations: CI, confidence intervals; CIMP, CpG island methylator phenotype; OR, odds ratio; WT, wild type.

The statistically significant $P$-values have been highlighted in bold.

A reduction in, or loss of, CDX2 staining was associated with the positive expression of MUC2, MUC5AC, and MUC6, whereas no significant association was observed for MUC5B. However, when MUC5B expression was considered in terms of high ( $>50 \%$ tumor cells positive) or low MUC5B expression, there was a stronger inverse effect with reduction in, or loss of, CDX2 staining, although this did not achieve statistical significance $(\mathrm{OR}=2.75,95 \%$ $\mathrm{CI}=0.97-7.81)$. Similarly, when MUC2 expression was classified as high expression ( $>50 \%$ positive tumor cells) and low expression, a stronger effect was observed with CDX2 deficiency $(\mathrm{OR}=5.10$, $95 \% \mathrm{CI}=1.86-14.08)$ than with a MUC2-positive staining threshold of $25 \% \quad(\mathrm{OR}=3.05, \quad 95 \%$ $\mathrm{CI}=1.13-8.26)$. Reduced or complete loss of expression of CDX2 was associated with CIMP positivity $(\mathrm{OR}=5.16,95 \% \mathrm{CI}=1.20-22.4)$, the $B R A F \mathrm{p} . \mathrm{V} 600 \mathrm{E}$ mutation $(\mathrm{OR}=5.05,95 \% \mathrm{CI}=1.73-14.73), M L H 1$ methylation ( $\mathrm{OR}=14.55,95 \% \mathrm{CI}=2.50-84.56)$, and mismatch repair deficiency $(\mathrm{OR}=5.93,95 \% \mathrm{CI}=$ 2.04-17.26), but not KRAS mutation $(\mathrm{OR}=0.78$, $95 \%$ CI $=0.26-2.34)$. An example of a CDX2deficient colorectal carcinoma with expression of all four mucins is shown in Figure 5.

\section{Discussion}

We assessed expression of epithelial mucins MUC2, MUC5AC, MUC5B, and MUC6 in a large series of colorectal carcinomas, and related it to tumor clinicopathological features. We observed ectopic expression of the gastric mucins MUC5AC and MUC6 in $49 \%$ and $39 \%$ of tumors, respectively, and overexpression of the colonic mucins MUC2 and MUC5B in $33 \%$ and $48 \%$ of cancers, respectively. There were strong associations between
MUC2, MUC5AC, and MUC6 expression and carcinomas that demonstrate CIMP positivity, BRAF p.V600E mutation, MLH1 promoter methylation, mismatch repair deficiency, tumor-infiltrating lymphocytes, and proximal location, all of which are features associated with the CIMP-positive subtype of colorectal carcinoma ${ }^{3}$ and the serrated neoplasia tumorigenic pathway. In the most comprehensive study of mucin protein expression in colorectal cancer, we have also shown that overexpression of one mucin protein is frequently accompanied by the co-expression of one or more of the other mucins whose genes reside on chromosome 11p15.5, suggesting that a tumor milieu exists, which favors expression of these mucin genes. We have also shown an inverse association between the reduced or absent expression of a putative mucin gene regulator, CDX2, and positive expression of the MUC2, MUC5AC, and MUC6 proteins in colorectal cancer.

Of the chromosome 11 mucins, MUC2 expression has been most extensively studied in colorectal malignancy where it is commonly downregulated both at the mRNA and protein levels, ${ }^{16,38,39}$ except in tumors displaying a mucinous phenotype. ${ }^{19,20,40}$ Various immunohistochemical studies have reported MUC2 positivity in colorectal cancers ranging from 21 to $63 \% .^{19,39,41-43}$ MUC5AC expression has been reported in $13-64 \%$ carcinomas, ${ }^{16,42,44,45}$ with highest levels of expression in microsatellite-unstable/mismatch repair-deficient tumors ${ }^{18,19,46}$ and those showing characteristic mucinous phenotype. ${ }^{19,47}$ MUC5B and MUC6 expression in the lower gastrointestinal tract has remained relatively unexplored. MUC5B has been detected in normal colon where expression appears to be restricted to goblet cells, ${ }^{15,48}$ and in the only study to date, MUC5B expression in 3/8 colorectal cancers was diffusely and strongly positive. ${ }^{44}$ 

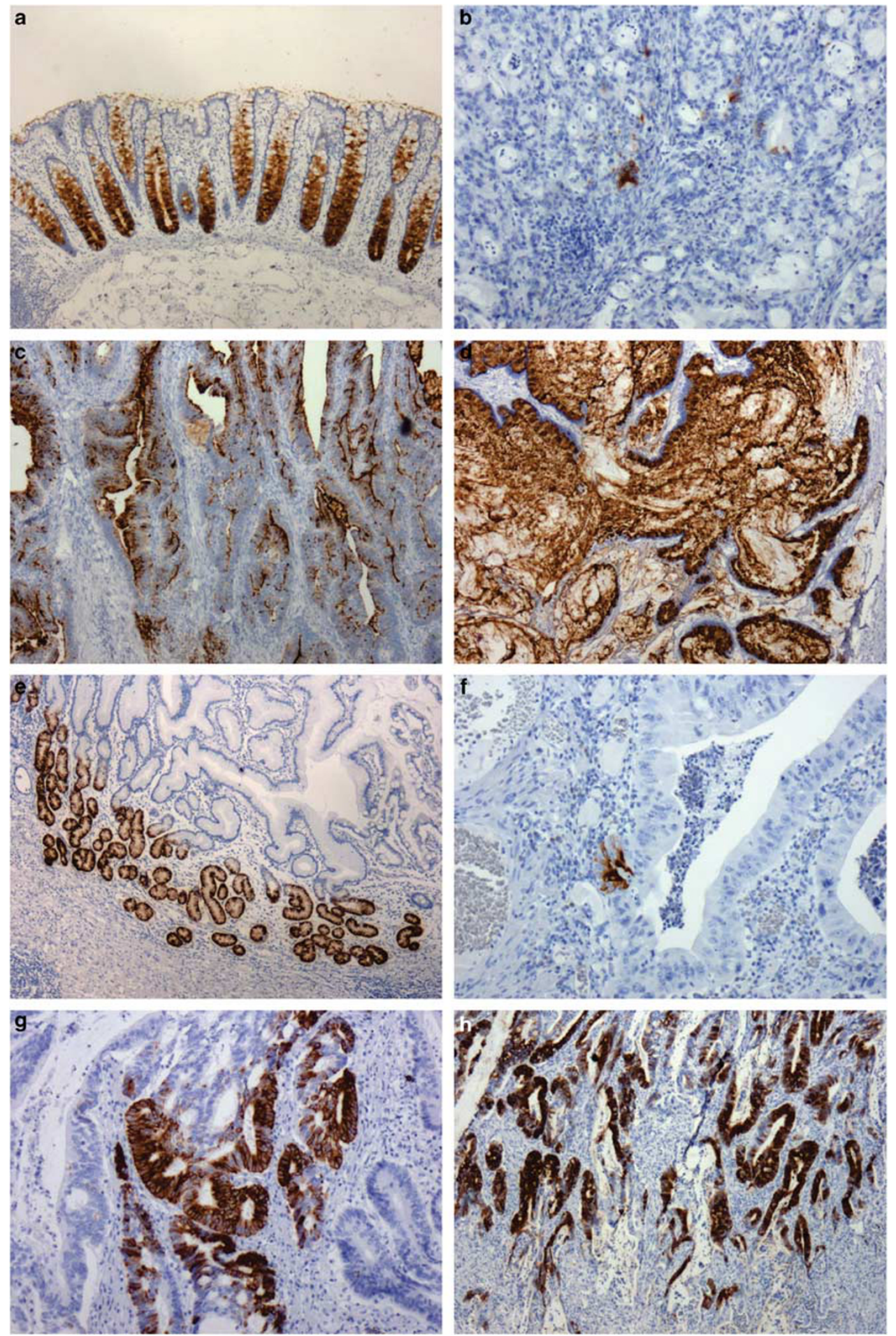

Figure 3 (a) MUC5B expression in normal colon shows a gradient along the crypt, being most intense in the crypt bases. Staining is present in both goblet cells and absorptive cells. (b) Rare isolated MUC5B-positive tumor cells within a negative colorectal carcinoma. (c) Moderate MUC5B staining of a conventional adenocarcinoma without evidence of mucinous differentiation. (d) Mucinous carcinoma showing intense MUC5B reactivity in both the epithelial cells and extracellular mucin. (e) MUC6 reactivity in the normal stomach is restricted to the gastric glands. (f) Rare intensely MUC6-positive tumor cells. (g) Approximately 50\% of tumor cells stained in a conventional adenocarcinoma without mucinous differentiation. (h) Diffuse MUC6 immunoreactivity in a mucinous cancer. 
Table 3 Reduced or absent CDX2 expression compared with clinicopathological features and mucin expression

\begin{tabular}{|c|c|c|c|}
\hline & $\mathrm{N}(\%)$ & $\mathrm{P}$ & OR $(95 \%$ CI) \\
\hline \multicolumn{4}{|l|}{ Tumor side } \\
\hline Right & $13 / 47(35.4 \%)$ & & \\
\hline Left & 7/69 (10.1\%) & 0.023 & $3.39(1.23-9.29)$ \\
\hline \multicolumn{4}{|l|}{$B R A F$} \\
\hline WT & 12/86 (14.0\%) & & \\
\hline p.V600E & $9 / 20(45.0 \%)$ & 0.004 & $5.05(1.73-14.73)$ \\
\hline \multicolumn{4}{|l|}{ MLH1 methylation } \\
\hline Unmethylated & $11 / 75(14.7 \%)$ & & \\
\hline Methylated & $5 / 7(71.4 \%)$ & 0.003 & $14.55(2.50-84.56)$ \\
\hline \multicolumn{4}{|l|}{ KRAS } \\
\hline WT & $16 / 78(20.5 \%)$ & & \\
\hline Mutant & $5 / 30(16.7 \%)$ & 0.789 & $0.78(0.26-2.34)$ \\
\hline \multicolumn{4}{|l|}{ CIMP } \\
\hline Negative & 11/82 (13.4\%) & & \\
\hline Positive & $4 / 9(44.4 \%)$ & 0.038 & $5.16(1.20-22.24)$ \\
\hline \multicolumn{4}{|l|}{ Mismatch repair } \\
\hline Proficient & $12 / 99(12.1 \%)$ & & \\
\hline Deficient & $9 / 20(45.0 \%)$ & 0.002 & $5.93(2.04-17.26)$ \\
\hline \multicolumn{4}{|l|}{ MUC2 } \\
\hline Negative & 7/65 (10.8\%) & & \\
\hline Positive & $14 / 52(26.9 \%)$ & 0.030 & $3.05(1.13-8.26)$ \\
\hline \multicolumn{4}{|l|}{ MUC2 HIGH } \\
\hline Negative & $7 / 76(9.2 \%)$ & & \\
\hline Positive & $14 / 41(28.1 \%)$ & 0.002 & $5.10(1.86-14.08)$ \\
\hline \multicolumn{4}{|l|}{$M U C 5 A C$} \\
\hline Negative & $4 / 58(6.9 \%)$ & & \\
\hline Positive & $17 / 60(28.3 \%)$ & 0.003 & 5.35 (1.67-16.95) \\
\hline \multicolumn{4}{|l|}{ MUC6 } \\
\hline Negative & $6 / 70(8.6 \%)$ & & \\
\hline Positive & $14 / 43(32.6 \%)$ & 0.002 & $5.15(1.80-14.71)$ \\
\hline \multicolumn{4}{|l|}{$M U C 5 B$} \\
\hline Negative & $7 / 51(13.7 \%)$ & & \\
\hline Positive & $11 / 55(20.0 \%)$ & 0.445 & $1.57(0.56-4.24)$ \\
\hline \multicolumn{4}{|l|}{ MUC5B HIGH } \\
\hline Negative & 7/63 (11.1\%) & & \\
\hline Positive & $11 / 43(25.6 \%)$ & 0.067 & $2.75(0.97-7.81)$ \\
\hline
\end{tabular}

Abbreviations: CI, confidence intervals; CIMP, CpG island methylator phenotype; OR, odds ratio; WT, wild type.

The statistically significant $P$-values have been highlighted in bold.

Similarly, MUC6 expression in colorectal tumors has been restricted largely to adenomas and serrated polyps, the latter have been found to express MUC6 commonly, ${ }^{30,49}$ whereas several groups have reported MUC6 expression in cancers as rare or absent, ${ }^{16,50,51}$ even in mucinous carcinomas. ${ }^{52}$

In 1999, Toyota et $a l^{53}$ described the existence of the CIMP in colorectal carcinoma, in which hypermethylation is induced in a wide variety of genes. Ferracin et $a l^{9}$ found that CIMP-positive colorectal cancers were more likely than CIMPnegative cancers to show areas of mucinous

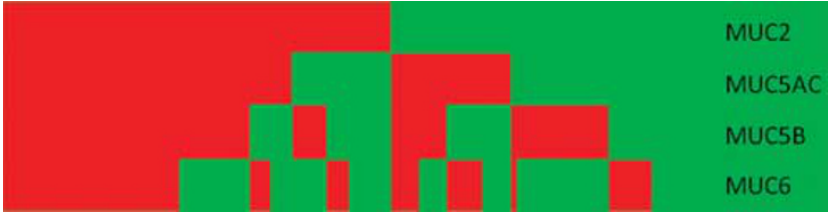

Figure 4 'Heat map' style representation of the co-expression of the four chromosome 11 mucins in 117 colorectal cancers. Positive tumors for each mucin are highlighted in red and negative cases are highlighted in green.

differentiation. Several studies have examined the expression of various mucin genes in relation to the nature and extent of $\mathrm{CpG}$ island methylation, and have found that MUC2 and MUC5B in particular, show strong correlations between promoter methylation and suppression of mucin protein synthesis. $^{23,54-57}$ There is currently little or no evidence of methylation-induced silencing of either MUC5AC or MUC6. ${ }^{23}$ Furthermore, MUC17, a transmembrane mucin gene, is regulated by promoter CpG island methylation and histone H3-K9 acetylation in pancreatic ductal adenocarcinomas, where promoter hypomethylation is associated with MUC17 expression. ${ }^{58}$ In this study, we have shown increased expression of MUC proteins in CIMPpositive colorectal cancers, which suggests the counter intuitive finding of increased protein expression in an environment that methylates (and silences) gene promoters. Interestingly, Ferracin et $a l^{9}$ found similar evidence of upregulation of MUC17 mRNA in CIMP cancers, supporting the concept of alternate regulatory mechanisms besides promoter methylation as a cause of increased MUC protein expression.

Besides mucin gene promoter methylation, expression of the chromosome 11 mucins is reportedly controlled by a number of other regulatory systems, including the EGFR-RAS-RAF pathway and paracrine exposure to cytokines (reviewed by Adrianifahanana et $a l^{25}$ ). Several studies have reported a link between somatic mutations in components of the EGFR-RAS-RAF pathway and evidence of mucinous differentiation in colorectal carcinomas, ${ }^{59-63}$ but the inclusion of microsatelliteunstable carcinomas in many of these studies is likely to have confounded the association because of previous observations that microsatellite-unstable tumors are associated with overexpression of MUC2 and MUC5 $\mathrm{AC}^{19}$ and a mucinous phenotype. ${ }^{11,12,64}$ KRAS mutations have been identified in $65 \%$ of mucinous colorectal carcinoma, ${ }^{65}$ and associations between mutations in the EGFR-RAS-RAF pathway and other types of malignancy have been made, ${ }^{66}$ including mucinous ovarian cancers, half or more of which possess somatic KRAS mutations. ${ }^{67,68}$ Taken together, these studies implicate activation of the EGFR-RAS-RAF pathway in upregulating mucin synthesis. The results from our current study support the association between expression of MUC2, MUC5AC, and MUC6 and activation 

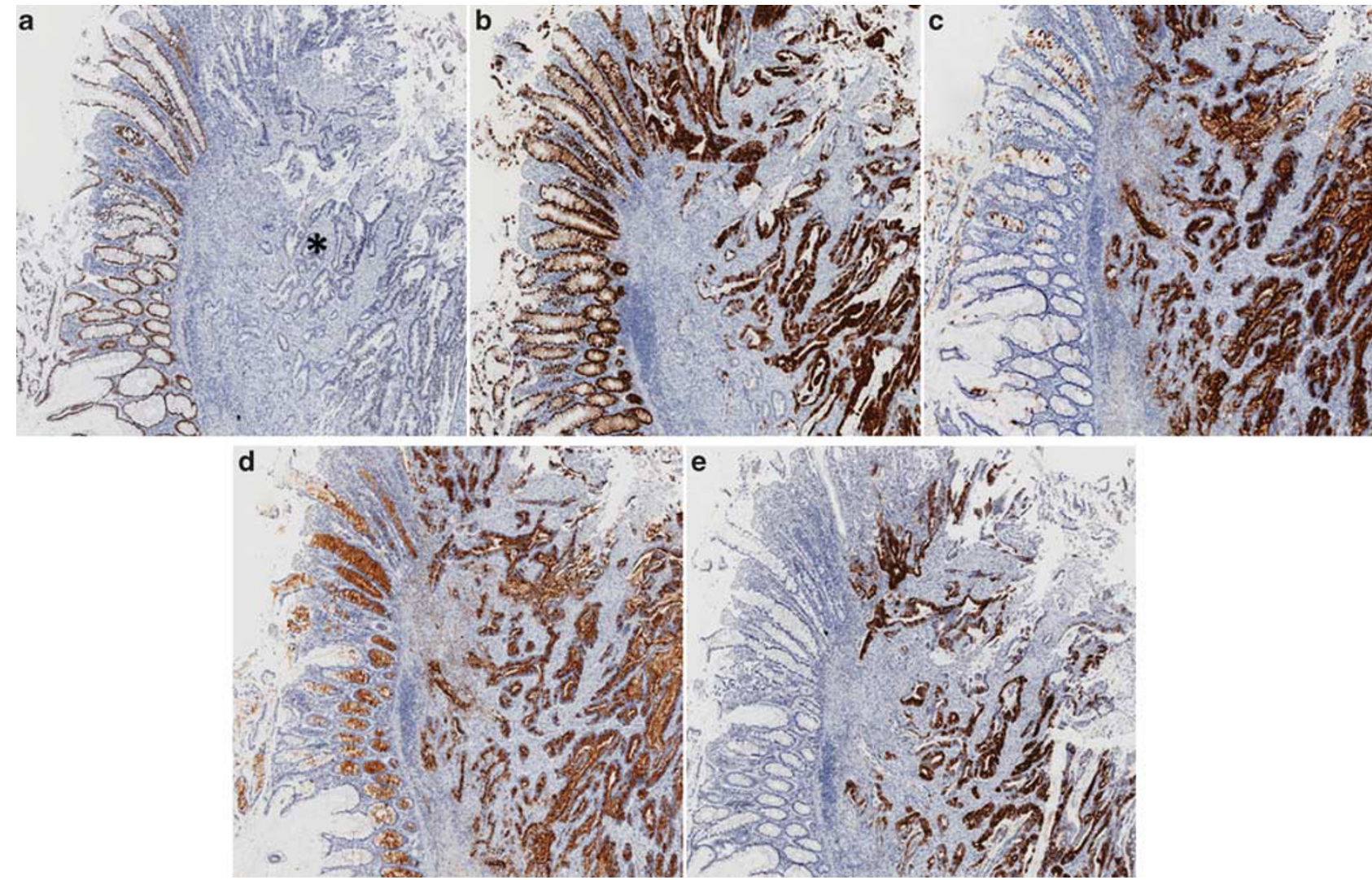

Figure 5 CDX2 loss associated with strong expression of all four chromosome 11 mucins. (a) CDX2 expression is present within the normal epithelium (left) but is significantly reduced in the colorectal cancer (*). (b) MUC2, (c) MUC5AC, (d) MUC5B, and (e) MUC6 expression in the same tumor.

of the EGFR-RAS-RAF pathway via the presence of somatic BRAF p.V600E mutation, with KRAS codon 12 and 13 mutations also associated with the overexpression of MUC2.

The mucin genes contain numerous binding sites for transcription factors, including Sp1, SP3, AP-1, various members of the GATA family, $\mathrm{NF} \kappa \mathrm{B}$, and CDX2 among others (reviewed in Adrianifahanana et $a l^{25}$ ). CDX2 has been identified by Yamamoto et $a I^{27}$ as a protein able to bind to the MUC2 promoter and initiate transcription, and subsequent studies by Mesquita et al have shown two functionally active CDX2-binding sites. ${ }^{26}$ Ectopic expression of CDX2 has also been linked to MUC2 expression in gastric intestinal metaplasia, ${ }^{69}$ Barrett's esophagus, ${ }^{70}$ and cholangiocarcinoma, ${ }^{71}$ suggesting an important regulatory role for CDX2 in MUC2 expression. We and others have reported that CDX2 may be somatically lost in some colorectal cancers either due to out-offrame allelic gains or losses of the microsatellite repeat within the $C D X 2$-coding region, ${ }^{29,72}$ epigenetic silencing via methylation, ${ }^{73}$ or loss of heterozygosity. ${ }^{74}$ Interestingly, Mochizuka et al ${ }^{30}$ reported reduced expression or complete loss of CDX2 in serrated polyps of the colorectum in combination with increased expression of MUC2, MUC5AC, and MUC6.
We have found that CDX2 expression was completely lost in $5 \%$ of colorectal carcinoma cases and noticeably reduced in a further $12.5 \%$. There was a strong inverse relationship between loss of expression of CDX2 and the overexpression of mucins MUC2, MUC5AC, and MUC6. Loss of CDX2 was also strongly linked to CIMP, MLH1 methylation, and BRAF mutation, as well as mismatch repair deficiency in these tumors, features that were also associated with MUC2, MUC5AC, and MUC6 overexpression. In our current study design, it is difficult to determine whether the associations observed between MUC protein expression, CDX2 expression and features of the serrated pathway of tumorigenesis (CIMP, BRAF mutation, and MLH1 methylation) are a cause or a consequence of altered MUC protein expression. Serrated colorectal polyps have been shown to have high levels of methylation and somatic BRAF mutations, and are thought to be the precursor lesions for many non-familial microsatellite-unstable cancers in which MLH1 has been epigenetically silenced. ${ }^{75}$ Our observations are, thus, in keeping with the findings of Mochizuka et al. ${ }^{30}$ Although CDX2 might have an important role in regulating MUC2 expression, particularly in the normal colorectum, the findings of increased expression of MUC2 (and other chromosome 
11 mucins) in a setting of decreased or lost expression of CDX2 suggest that other regulatory mechanisms are likely to be involved to compensate for the loss of CDX2.

Previous studies have described mucinous differentiation based solely on morphological assessment rather than mucin protein expression, which precludes detecting mucin gene overexpression in tumors lacking a component of characteristic mucinous differentiation. We have shown that microsatellite-unstable colorectal carcinomas lacking mucinous or signet ring cell differentiation overexpress one or more chromosome 11 mucin proteins, and that overexpression is often linked to the presence of the activating BRAF p.V600E mutation in these cancers.

In conclusion, we report the expression pattern of chromosome 11 gel-forming mucins, MUC2, MUC5AC, MUC5B, and MUC6 in a large series of colorectal carcinomas and, in doing so, provide the first comprehensive immunohistochemical assessment of MUC5B expression in colorectal tumors. We have demonstrated heterogeneity of expression of these mucins, such that overexpression was strongly associated with features associated with the CIMP subtype of colorectal cancer, including the $B R A F$ p.V600E somatic mutation, mismatch repair deficiency, and proximal tumor location. In addition, we provide evidence for the role of the homeobox gene CDX2 in mucin expression, demonstrating an inverse association with the expression of the chromosome 11 gel-forming mucins, suggesting that additional mucin regulatory mechanisms are altered in carcinomas that overexpress these mucins.

\section{Acknowledgements}

We thank Charmaine Smith, Lisa Oates, and Sonia Terre'Blanche from the Cancer Council Victoria for their tireless assistance with tissue block acquisition, Diane McKeone and Erika Pavluk for technical and database assistance, the late Professor Jeremy Jass for performing some of the histopathology reviews, and Dr Karine Rousseau (University of Manchester, UK) for her gift of the MUC5B monoclonal antibody used in the study. The authors are also indebted to Heather Matthews for providing graphics support. We would like to thank the various clinical laboratories that very kindly made access to tissue blocks possible. This study was supported by a grant from the NHMRC. Christophe Rosty is the Jass Pathology Fellow, John L Hopper is an NMHRC Australia Fellow, and Mark A Jenkins and Michael A McGuckin are supported by NHMRC Senior Research Fellowships. MCCS recruitment was funded by VicHealth, and ongoing support has been provided from recurrent funding by Cancer Council Victoria. This work was funded in part by a NHMRC Program Grant (APP209057).

\section{Disclosure/conflict of interest}

The authors declare no conflict of interest.

\section{References}

1 Vogelstein B, Fearon ER, Kern SE, et al. Allelotype of colorectal carcinomas. Science 1989;244:207-211.

2 Toyota M, Ahuja N, Suzuki H, et al. Aberrant methylation in gastric cancer associated with the $\mathrm{CpG}$ island methylator phenotype. Cancer Res 1999;59:5438-5442.

3 Weisenberger DJ, Siegmund KD, Campan M, et al. CpG island methylator phenotype underlies sporadic microsatellite instability and is tightly associated with BRAF mutation in colorectal cancer. Nat Genet 2006;38:787-793.

4 Kambara T, Simms LA, Whitehall VL, et al. BRAF mutation is associated with DNA methylation in serrated polyps and cancers of the colorectum. Gut 2004;53:1137-1144.

5 Nagasaka T, Koi M, Kloor M, et al. Mutations in both KRAS and BRAF may contribute to the methylator phenotype in colon cancer. Gastroenterol 2008; 134:1950-1960.

6 Ogino S, Brahmandam M, Kawasaki T, et al. Combined analysis of COX-2 and p53 expressions reveals synergistic inverse correlations with microsatellite instability and $\mathrm{CpG}$ island methylator phenotype in colorectal cancer. Neoplasia 2006;8:458-464.

7 Nosho K, Irahara N, Shima K, et al. Comprehensive biostatistical analysis of $\mathrm{CpG}$ island methylator phenotype in colorectal cancer using a large population-based sample. PLoS ONE 2008;3:e3698.

$8 \mathrm{Li} \mathrm{WQ}$, Kawakami K, Ruszkiewicz A, et al. BRAF mutations are associated with distinctive clinical, pathological and molecular features of colorectal cancer independently of microsatellite instability status. Mol Cancer 2006;5:2.

9 Ferracin M, Gafa R, Miotto E, et al. The methylator phenotype in microsatellite stable colorectal cancers is characterized by a distinct gene expression profile. J Pathol 2008;214:594-602.

10 Samowitz WS, Albertsen H, Herrick J, et al. Evaluation of a large, population-based sample supports a CpG island methylator phenotype in colon cancer. Gastroenterol 2005;129:837-845.

11 Jass JR, Do KA, Simms LA, et al. Morphology of sporadic colorectal cancer with DNA replication errors. Gut 1998:42:673-679.

12 Shia J, Ellis NA, Paty PB, et al. Value of histopathology in predicting microsatellite instability in hereditary nonpolyposis colorectal cancer and sporadic colorectal cancer. Am J Surg Pathol 2003;27:1407-1417.

13 Pigny P, Guyonnet-Duperat V, Hill AS, et al. Human mucin genes assigned to 11 p15.5 - identification and organization of a cluster of genes. Genomics 1996;38:340-352.

14 Winterford CM, Walsh MD, Leggett BA, et al. Ultrastructural localization of epithelial mucin core proteins in colorectal tissues. J Histochem Cytochem 1999;47:1063-1074.

15 van Klinken BJW, Dekker J, van Gool SA, et al. MUC5B is the prominent mucin in human gallbladder and is also expressed in a subset of colonic goblet cells. Am J Physiol Gastrointest Liver Physiol 1998;37: G871-G878. 
16 Sylvester PA, Myerscough $\mathrm{N}$, Warren $\mathrm{BF}$, et al. Differential expression of the chromosome 11 mucin genes in colorectal cancer. J Pathol 2001;195: 327-335.

17 de Bolós C, Garrido M, Real FX. MUC6 apomucin shows a distinct normal tissue distribution that correlates with Lewis antigen expression in the human stomach [comment]. Gastroenterol 1995;109:723-734.

18 Losi L, Scarselli A, Benatti P, et al. Relationship between MUC5AC and altered expression of MLH1 protein in mucinous and non-mucinous colorectal carcinomas. Pathol Res Pract 2004;200:371-377.

19 Biemer-Hüttmann AE, Walsh MD, McGuckin MA, et al. Mucin core protein expression in colorectal cancers with high levels of microsatellite instability indicates a novel pathway of morphogenesis. Clin Cancer Res 2000;6:1909-1916.

20 Arai T, Kasahara I, Sawabe M, et al. Microsatelliteunstable mucinous colorectal carcinoma occurring in the elderly: comparison with medullary type poorly differentiated adenocarcinoma. Pathol Int 2007;57:205-212.

21 Park SY, Lee HS, Choe G, et al. Clinicopathological characteristics, microsatellite instability, and expression of mucin core proteins and p53 in colorectal mucinous adenocarcinomas in relation to location. Virchows Arch 2006;449:40-47.

22 Hanski C, Riede E, Gratchev A, et al. MUC2 gene suppression in human colorectal carcinomas and their metastases: In vitro evidence of the modulatory role of DNA methylation. Lab Invest 1997;77:685-695.

23 Vincent A, Perrais M, Desseyn JL, et al. Epigenetic regulation (DNA methylation, histone modifications) of the 11p15 mucin genes (MUC2, MUC5AC, MUC5B, MUC6) in epithelial cancer cells. Oncogene 2007;26:6566-6576.

24 Perrais M, Pigny $\mathrm{P}$, Copin MC, et al. Induction of MUC2 and MUC5AC mucins by factors of the epidermal growth factor (EGF) family is mediated by EGF receptor/Ras/Raf/extracellular signal-regulated kinase cascade and Sp1. J Biol Chem 2002;277: 32258-32267.

25 Andrianifahanana M, Moniaux N, Batra SK. Regulation of mucin expression: mechanistic aspects and implications for cancer and inflammatory diseases. Biochim Biophys Acta 2006;1765:189-222.

26 Mesquita P, Jonckheere N, Almeida R, et al. Human MUC2 mucin gene is transcriptionally regulated by $\mathrm{Cdx}$ homeodomain proteins in gastrointestinal carcinoma cell lines. J Biol Chem 2003;278: 51549-51556.

27 Yamamoto H, Bai YQ, Yuasa Y. Homeodomain protein CDX2 regulates goblet-specific MUC2 gene expression. Biochem Biophys Res Commun 2003;300:813-818.

28 Hinoi T, Tani M, Lucas PC, et al. Loss of CDX2 expression and microsatellite instability are prominent features of large cell minimally differentiated carcinomas of the colon. Am J Pathol 2001;159: 2239-2248.

29 Wicking C, Simms LA, Evans T, et al. CDX2, a human homologue of Drosophila caudal, is mutated in both alleles in a replication error positive colorectal cancer. Oncogene 1998;17:657-659.

30 Mochizuka A, Uehara T, Nakamura T, et al. Hyperplastic polyps and sessile serrated 'adenomas' of the colon and rectum display gastric pyloric differentiation. Histochem Cell Biol 2007;128:445-455.
31 Giles GG. The Melbourne Collaborative Cohort Study. IARC Sci Publ 2002;156:69-70.

32 Ueno H, Murphy J, Jass JR, et al. Tumour 'budding' as an index to estimate the potential of aggressiveness in rectal cancer. Histopathol 2002;40:127-132.

33 Young J, Simms LA, Biden KG, et al. Features of colorectal cancers with high-level microsatellite instability occurring in familial and sporadic settings: parallel pathways of tumorigenesis. Am J Pathol 2001;159:2107-2116.

34 Fritz A, Percy C, Jack A, et al. International Classification of Diseases for Oncology (ICD-O), 3rd edn. World Health Organization: Geneva, 2000.

35 Walsh MD, Cummings MC, Buchanan DD, et al. Molecular, pathologic, and clinical features of earlyonset endometrial cancer: identifying presumptive lynch syndrome patients. Clin Cancer Res 2008; 14:1692-1700.

36 Lindor NM, Burgart LJ, Leontovich O, et al. Immunohistochemistry versus microsatellite instability testing in phenotyping colorectal tumors. J Clin Oncol 2002; 20:1043-1048.

37 English DR, Young JP, Simpson JA, et al. Ethnicity and risk for colorectal cancers showing somatic BRAF V600E mutation or CpG island methylator phenotype. Cancer Epidemiol Biomarkers Prev 2008;17: 1774-1780.

38 Weiss AA, Babyatsky MW, Ogata S, et al. Expression of MUC2 and MUC3 mRNA in human normal, malignant, and inflammatory intestinal tissues. J Histochem Cytochem 1996;44:1161-1166.

39 Blank M, Klussmann E, Kruger-Krasagakes S, et al. Expression of MUC2-mucin in colorectal adenomas and carcinomas of different histological types. Int J Cancer 1994;59:301-306.

40 Hanski C, Hofmeier M, Schmitt-Graff A, et al. Overexpression or ectopic expression of MUC2 is the common property of mucinous carcinomas of the colon, pancreas, breast, and ovary. J Pathol 1997;182:385-391.

41 Baldus SE, Monig SP, Hanisch FG, et al. Comparative evaluation of the prognostic value of MUC1, MUC2, sialyl-Lewis(a) and sialyl-Lewis(x) antigens in colorectal adenocarcinoma. Histopathol 2002;40:440-449.

42 Lau SK, Weiss LM, Chu PG. Differential expression of MUC1, MUC2, and MUC5AC in carcinomas of various sites: an immunohistochemical study. Am J Clin Pathol 2004;122:61-69.

$43 \mathrm{Bu} \mathrm{XD,} \mathrm{Li} \mathrm{N,} \mathrm{Tian} \mathrm{XQ,} \mathrm{et} \mathrm{al.} \mathrm{Altered} \mathrm{expression} \mathrm{of}$ MUC2 and MUC5AC in progression of colorectal carcinoma. World J Gastroenterol 2010;16:4089-4094.

44 Zhang S, Zhang HS, Cordon-Cardo C, et al. Selection of tumor antigens as targets for immune attack using immunohistochemistry: protein antigens. Clin Cancer Res 1998;4:2669-2676.

$45 \mathrm{Yu}$ XW, Rong W, Xu FL, et al. Expression and clinical significance of mucin and E-cadherin in colorectal tumors. Ai Zheng 2007;26:1204-1210.

$46 \mathrm{Li} \mathrm{D,} \mathrm{Semba} \mathrm{S,} \mathrm{Wu} \mathrm{M,} \mathrm{et} \mathrm{al.} \mathrm{Molecular} \mathrm{pathological}$ subclassification of mucinous adenocarcinoma of the colorectum. Pathol Int 2005;55:766-774.

47 Melis M, Hernandez J, Siegel EM, et al. Gene expression profiling of colorectal mucinous adenocarcinomas. Dis Colon Rectum 2010;53:936-943.

48 Carrato C, Balague C, de Bolos C, et al. Differential apomucin expression in normal and neoplastic human gastrointestinal tissues. Gastroenterol 1994;107: $160-172$. 
49 Fujita K, Hirahashi M, Yamamoto H, et al. Mucin core protein expression in serrated polyps of the large intestine. Virchows Arch 2010;457:443-449.

50 Nakajima K, Ota H, Zhang MX, et al. Expression of gastric gland mucous cell-type mucin in normal and neoplastic human tissues. J Histochem Cytochem 2003;51:1689-1698.

51 Matsuda M, Sentani K, Noguchi T, et al. Immunohistochemical analysis of colorectal cancer with gastric phenotype: claudin-18 is associated with poor prognosis. Pathol Int 2010;60:673-680.

52 Onodera M, Nishigami T, Torii I, et al. Comparison between colorectal low- and high-grade mucinous adenocarcinoma with MUC1 and MUC5AC. World J Gastrointest Oncol 2009;1:69-73.

53 Toyota M, Ahuja N, Ohe-Toyota M, et al. CpG island methylator phenotype in colorectal cancer. Proc Natl Acad Sci USA 1999;96:8681-8686.

54 Yamada N, Hamada T, Goto M, et al. MUC2 expression is regulated by histone $\mathrm{H} 3$ modification and DNA methylation in pancreatic cancer. Int J Cancer 2006;119:1850-1857.

55 Perrais M, Pigny $\mathrm{P}$, Buisine MP, et al. Aberrant expression of human mucin gene MUC5B in gastric carcinoma and cancer cells. Identification and regulation of a distal promoter. J Biol Chem 2001;276:15386-15396.

56 Mesquita P, Peixoto AJ, Seruca R, et al. Role of sitespecific promoter hypomethylation in aberrant MUC2 mucin expression in mucinous gastric carcinomas. Cancer Lett 2003;189:129-136.

57 Okudaira K, Kakar S, Cun L, et al. MUC2 gene promoter methylation in mucinous and non-mucinous colorectal cancer tissues. Int J Oncol 2010;36:765-775.

58 Kitamoto S, Yamada N, Yokoyama S, et al. DNA methylation and histone H3-K9 modifications contribute to MUC17 expression. Glycobiology 2011;21: 247-256.

59 Yokota T, Ura T, Shibata N, et al. BRAF mutation is a powerful prognostic factor in advanced and recurrent colorectal cancer. Br J Cancer 2011;104:856-862.

60 Chang SC, Lin JK, Yang SH, et al. Relationship between genetic alterations and prognosis in sporadic colorectal cancer. Int J Cancer 2006;118:1721-1727.

61 Tanaka H, Deng G, Matsuzaki K, et al. BRAF mutation, CpG island methylator phenotype and microsatellite instability occur more frequently and concordantly in mucinous than non-mucinous colorectal cancer. Int J Cancer 2006;118:2765-2771.

62 Ogino S, Brahmandam M, Cantor $\mathrm{M}$, et al. Distinct molecular features of colorectal carcinoma with signet ring cell component and colorectal carcinoma with mucinous component. Mod Pathol 2006;19:59-68.

$63 \mathrm{Li} \mathrm{WQ}$, Kawakami K, Ruszkiewicz A, et al. BRAF mutations are associated with distinctive clinical, pathological and molecular features of colorectal cancer independently of microsatellite instability status. Mol Cancer 2006;5:2.

64 Wright CL, Stewart ID. Histopathology and mismatch repair status of 458 consecutive colorectal carcinomas. Am J Surg Pathol 2003;27:1393-1406.

65 Laurent-Puig P, Olschwang S, Delattre O, et al. Association of Ki-ras mutation with differentiation and tumor-formation pathways in colorectal carcinoma. Int J Cancer 1991;49:220-223.

66 De Oliveira Duarte Achcar R, Nikiforova MN, Yousem SA. Micropapillary lung adenocarcinoma: EGFR, K-ras, and BRAF mutational profile. Am J Clin Pathol 2009;131:694-700.

67 Gemignani ML, Schlaerth AC, Bogomolniy F, et al. Role of KRAS and BRAF gene mutations in mucinous ovarian carcinoma. Gynecol Oncol 2003;90:378-381.

68 Enomoto T, Weghorst CM, Inoue M, et al. K-ras activation occurs frequently in mucinous adenocarcinomas and rarely in other common epithelial tumors of the human ovary. Am J Pathol 1991;139:777-785.

69 Almeida R, Silva E, Santos-Silva F, et al. Expression of intestine-specific transcription factors, CDX1 and CDX2, in intestinal metaplasia and gastric carcinomas. J Pathol 2003;199:36-40.

$70 \mathrm{Hu} \mathrm{Y}$, Jones C, Gellersen O, et al. Pathogenesis of Barrett esophagus: deoxycholic acid up-regulates goblet-specific gene MUC2 in concert with CDX2 in human esophageal cells. Arch Surg 2007;142:540-544.

71 Jinawath A, Akiyama Y, Yuasa Y, et al. Expression of phosphorylated ERK1/2 and homeodomain protein CDX2 in cholangiocarcinoma. J Cancer Res Clin Oncol 2006;132:805-810.

72 Hinoi T, Tani M, Lucas PC, et al. Loss of CDX2 expression and microsatellite instability are prominent features of large cell minimally differentiated carcinomas of the colon. Am J Pathol 2001;159: 2239-2248.

73 Kawai H, Tomii K, Toyooka S, et al. Promoter methylation downregulates CDX2 expression in colorectal carcinomas. Oncol Rep 2005;13:547-551.

74 Sivagnanasundaram S, Islam I, Talbot I, et al. The homeobox gene CDX2 in colorectal carcinoma: a genetic analysis. Br J Cancer 2001;84:218-225.

75 Spring KJ, Zhao ZZ, Karamatic R, et al. High prevalence of sessile serrated adenomas with BRAF mutations: a prospective study of patients undergoing colonoscopy. Gastroenterol 2006;131:1400-1407.

Supplementary Information accompanies the paper on Modern Pathology website (http://www.nature.com/ modpathol) 